\title{
Recommendations for actions concerning supporting ITS developments for VRUs
}

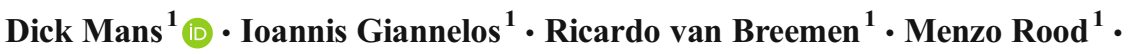 \\ Anne Silla ${ }^{2}$ Pirkko Rämä ${ }^{2} \cdot$ Johan Scholliers $^{3} \cdot$ Oscar Martin Perez $^{4} \cdot$ Kerry Malone $^{5}$
}

Received: 12 April 2016/Accepted: 21 March 2017 /Published online: 18 April 2017

(C) The Author(s) 2017. This article is an open access publication

\begin{abstract}
Introduction This paper presents some of the work conducted in FP7 VRUITS project to draw a set of general recommendations for actions at the EU level aiming to enhance the deployment of ITS systems directed to improve the safety, mobility and comfort of vulnerable road users.

Method The work started by identifying potential barriers, as well as recommendations to overcome these barriers. After that, similar recommendations were combined and prioritised, which are discussed via a workshop and questionnaire. In addition, a qualitative analysis was used to select thirteen main recommendations, for which a quantitative Benefit Analyses and a Multi-Criteria analysis were conducted.

Results In total, over 200 barriers and recommendations were identified for the 10 selected ITS systems. After several steps 13 main general recommendations were selected and ranked according to priority.

Conclusions 1) A wide range of different type of measures directed to different stakeholders is needed 2) Focus should be on facilitating the implementation of the 13 main
\end{abstract}

This article is part of Topical collection on The Influence of Intelligent Transport Systems on Vulnerable Road User Accidents

Dick Mans

dick.mans@ecorys.com

Ecorys, Watermanweg 44, 3067GG Rotterdam, the Netherlands

2 VTT, Vuorimiehentie 3, 02044 Espoo, Uusimaa FI, Finland

3 VTT, Tekniikankatu 1, 33100 Tampere, Finland

4 Cidaut, Parque Tecnológico de Boecillo, Plaza Vicente Aleixandre Campos 2, 47151 Boecillo, Valladolid, Spain

5 TNO, New Babylon. Anna van Buerenplein 1, 2595DA The Hague, the Netherlands recommendations, 3) Benefits of implementation of the recommendations can be in the order of billion(s) of euros, and 4) Main challenges are achieving cooperation between the different stakeholders, the different environments, the long time horizon for implementation and the high implementation costs.

Keywords Vulnerable road users · Intelligent transportation systems $\cdot$ Multi-criteria analysis $\cdot$ Human machine interface . Cooperative intelligent transportation systems

\section{Introduction}

Intelligent Transport Systems (ITS) are defined by [1] as advanced applications which, without embodying intelligence as such, aim to provide innovative systems relating to different modes of transport and traffic management and enable various users to be better informed and make safer, more coordinated and 'smarter' use of transport networks.

In recent years, both technological developments and research activities in the fields of ITS have primarily focussed on motorised transport to improve safety, mobility and ecological (environmental) impacts. In regard to safety, the uptake of ITS has led to the decrease of road traffic fatalities, particularly amongst passenger car occupants. However, Vulnerable Road Users (VRUs), such as pedestrians, cyclists, motorcyclists and moped riders have not experienced the same decrease in fatalities: inside European urban areas, pedestrian fatalities represent $39 \%$ of all fatalities [2].

The VRUITS project, which was co-sponsored by the European Commission through the FP7-programme, has identified [3] and analysed [4] a set of ITS systems, which have the potential to improve the safety and/or the mobility and comfort of VRUs. The selection of the systems was 
performed in two workshops with stakeholders. In the first workshop, the applications which were seen as having the most potential to improve safety and/or mobility and comfort were selected [3]. The assessment was performed in two steps: a qualitative and a quantitative step. It was based on the method developed by Kulmala [5] for vehicle safety systems, which uses a set of 9 mechanisms, via which ITS can affect road user behaviour and thereby road safety, which was adapted for VRUs [6]. A qualitative assessment of the impact on safety, mobility \& comfort was performed for a set of 23 systems [4]. In the second workshop with the stakeholders, the qualitative assessments were presented and a score was given by the participants to different aspects of the ITS systems (benefits, costs, deployment, user aspects). A multi-criteria analysis has been used to rank the different ITS systems. After that, a portfolio check was carried out to determine whether all important aspects were covered, for example, that systems addressed important vulnerable road user groups. This resulted in 10 ITS systems which were selected for a quantitative safety, mobility and comfort and socioeconomic assessment. The 10 systems selected for quantitative analysis are presented in Table 1.

Building on the results of the safety, mobility \& comfort assessment for 10 different ITS systems [4], and from the results of field trials on selected ITS systems [7], the VRUITS project has developed a set of recommendations for actions at the EU level regarding ITS systems for vulnerable road users and intelligent transportation systems,.

This paper presents the methodology to generate the general recommendations in Section 2. Section 3 presents the results for selecting and assessment of the general recommendations, while Section 4 presents the selected recommendations in more detail. The conclusions are outlined in Section 6.

Important sources of information for this paper are the VRUITS project deliverables. All these deliverables can be found at the VRUITS project website: www.vruits.eu.

\section{Table 1 Overview 10 selected ITS systems}

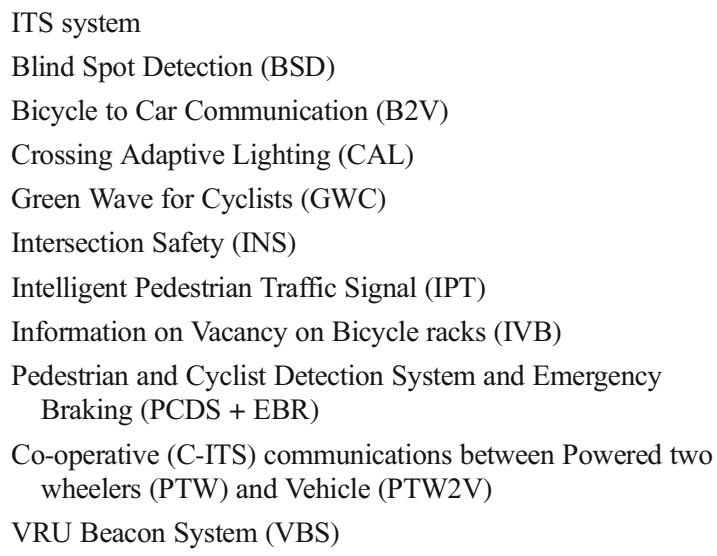

\section{Methodology general recommendations}

The methodology for the identification and ranking of the main recommendations to enable the maximum deployment of selected ITS systems in Europe, consisted of four main steps. These steps are presented in Fig. 1.

Each of these steps is described in more detail in the following:

1. The analysis of the 10 ITS systems consisted of an identification of potential barriers regarding the widespread use of the ITS systems in the EU-28, as well as recommendations to overcome these barriers. The analysis covered the following eight topics: "technical performance", "safety, mobility \& comfort effects", "human-machine interface (HMI)", "acceptance", "business models", "implementation issues", "legal and standardisation issues" and finally "privacy, data storage, ethical and moral issues". The identified recommendations were classified according to the following criteria (more details are provided in [8]):

- Ease of implementation: Easy, Medium, Challenging;

- Time horizon: Short term ( $<5$ years); Medium term (5-10 years) and Long term (>10 years);

- Estimated effectiveness: Low (does not completely remove the barrier), Medium (barrier is removed in most cases), High (barrier is completely removed). This classification was only valid if the recommendation could be linked to a specific barrier;

- Estimated costs: Low (hundred thousands of Euros); Medium (1-10 million Euros) and High (ten(s) of millions of Euros).

In addition, the relevant stakeholders were identified for each recommendation. A total of 213 barriers and 208 recommendations were identified. During the first analysis of recommendations, the recommendations were categorised in three different groups (recommendations regarding VRUs, vehicles and infrastructure). By means of grouping and additional analysis, the recommendations were merged and prioritised, resulting in a long list of 50 recommendations. A recommendation was included in the long list [8] if it scored high on the different criteria, such as having low implementation costs, being considered effective and concerning several motivated stakeholders.

2. Workshop and questionnaire. A workshop was held with the stakeholders in order to receive feedback and to prioritise the recommendations. A questionnaire was also 
Fig. 1 Overview methodology 'General recommendations'

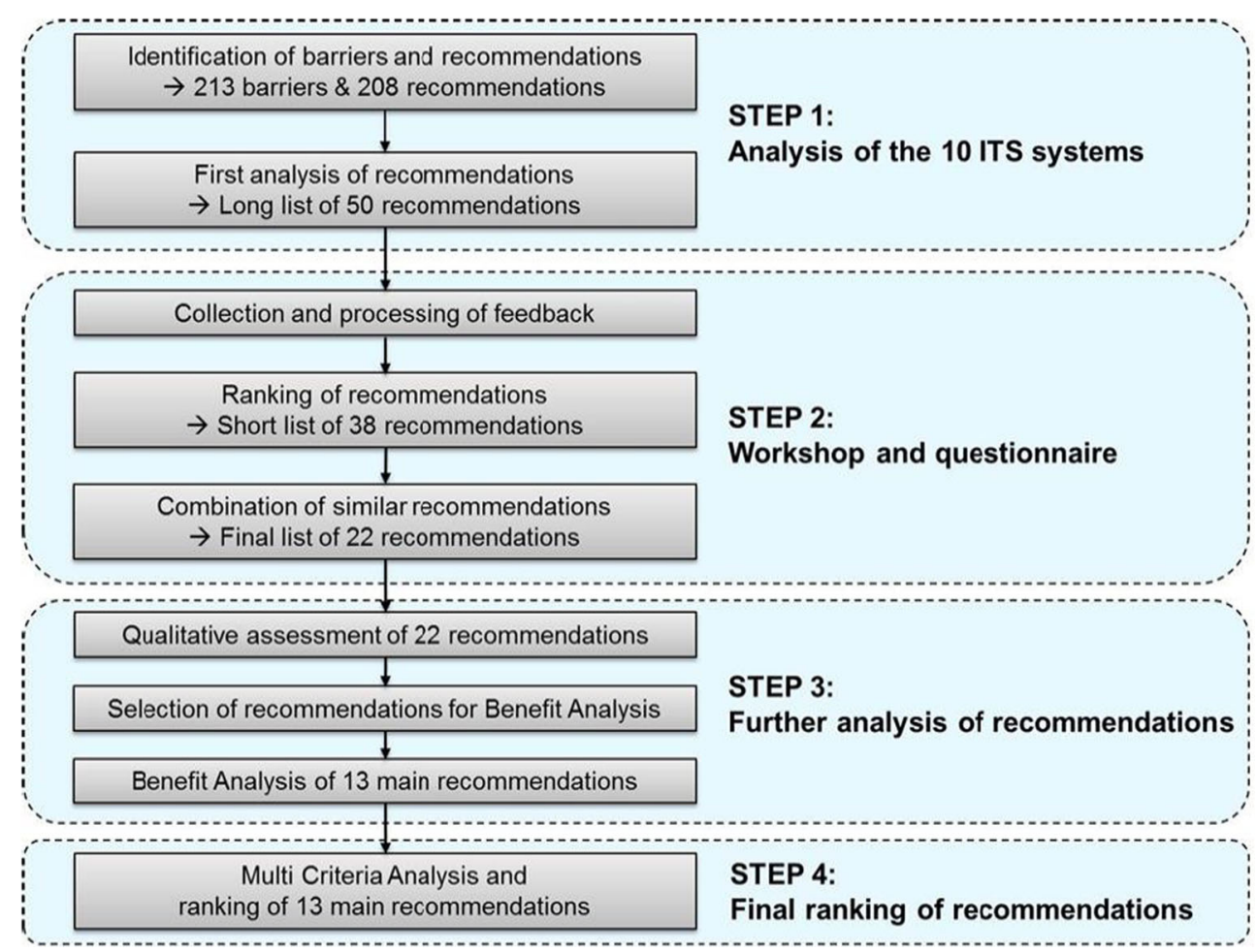

sent to relevant stakeholders who could not participate in the workshop, to verify the outcomes of the workshop and the selected recommendations. The process included the three following steps:

- Collection and processing the feedback of workshop participants. The recommendations were divided in three groups: recommendations regarding VRUs, vehicles and infrastructure. The workshop participants were divided into three groups and they participated in three different sessions, each covering one group of recommendations. The feedback collected during the workshop was used to update the contents and initial assessments of the presented recommendations.

- Ranking of recommendations. At the end of each session, the workshop participants were asked to indicate the five main recommendations in each group which they considered the most important to facilitate the effective market introduction of the systems at a European level. The participants were asked to use a scale from 1 to 5 with five points for the most important recommendations of each group, four for the second most important, etc. Based on the overall scores given by the workshop participants, the recommendations with an overall score of less than 5 points were not considered for further analysis. This resulted in a shortlist of 38 measures.

- Combination of similar measures. >In this phase, similar and/or overlapping recommendations were merged with each other. This resulted in a final list of 22 recommendations, which was verified by means of a questionnaire and sent to relevant stakeholders. The main purpose of the questionnaire was to outline the outcomes of the workshop and the selected recommendations. The respondents were asked to indicate if they agreed with the proposed recommendations, and if they saw any potential obstacles for the implementation of these recommendations. At the end, the respondents were requested to assess what they considered the most effective recommendation per topic.

3. Further analysis of recommendations. The analysis consisted of three main steps.

- Qualitative Analysis. An in-depth qualitative analysis of the 22 recommendations was performed on the criteria mentioned under 'Step 1'. This resulted in a more detailed description of the recommendations, identification of main implementation issues, description of roles and required actions of main stakeholders involved, more information about barriers addressed and description of main cost categories.

- Selection of 13 main recommendations. Based on the qualitative analysis together with the results of the workshop and the expert questionnaire, a list of 13 main recommendations was selected for a quantitative analysis of the potential benefits. 
- Benefit Analysis of 13 main recommendations. The method used for the Benefit Analysis was primarily based on the CBA framework used to assess the impacts of the 10 ITS systems in earlier phases of the VRUITS project [4]. The Benefit Analysis focussed on analysing the effects of carrying out the recommendation for each of these 10 ITS systems, compared to the situation where the recommendation would not be carried out. Each recommendation was considered to be effective for at least one of the 10 ITS systems. The effectiveness of each recommendation depended on the extent that the recommendation succeeds in removing the barrier. The recommendations are considered to directly enhance system performance in the areas of:

- Safety, e.g. number of fatalities, injuries;

- Comfort, e.g. comfort level;

- Mobility, e.g. trip length, number of trips, trip duration or modal shift;

- Penetration rates, e.g. percentage of potential users actually using the system; and

- System costs i.e. the investment costs.

The impact of the recommendations on system effectiveness was qualitatively assessed, based on expert judgement of the partners involved in this study, for each of the above-mentioned areas. The assessment was made by rating the effectiveness as according to the scale: i) no effect, ii) very small, iii) small, iv) average or v) large. A distinction was made in relation to effects which are relatively short-term (2020), and medium/long term (2030).

The main link between the qualitative assessment and the quantitative analysis is shown in Table 1 . The table details how the different impacts on system performance can be converted to parameter figures. Large impacts on effectiveness were matched to the most positive situation in the sensitivity analysis and scenario analysis, following the sensitivity analysis and scenarios from earlier VRUITS work [4]. For smaller impacts, the quantification was based on an interpolation between large impacts and no impacts, as shown in Table 2.

The assessment on safety, comfort and mobility was handled in the same way: an improvement in effectiveness resulted in higher benefits in the respective area, quantified by a percentage by which all effects related are multiplied. For example, if safety effectiveness is expected to have a small impact from the recommendation, the safety effectiveness parameter is $105 \%$. This was then used in the CBA calculations, multiplying the corresponding safety benefits with $105 \%$. Note that these percentages do not mean the system performs better than the theoretical maximum, but should be interpreted as an improvement to the theoretical maximum system performance.

The impact on the penetration rate was calculated differently. The original CBAs were based on penetration rate scenarios - scenarios that contain figures on the usage of the system. Higher usage of the system was assumed to increase the safety benefits. In addition, effects were also anticipated in mobility and comfort due to changes in the penetration rate. The scenarios were used to calculate the impact of the recommendation by taking the difference of the high scenario CBA results and the medium scenario $\mathrm{CBA}$ results. This difference was then multiplied by $1 / 8,1 / 4,1 / 2$ or 1 , depending on the assessment and Table 2 . The estimation of the recommendation benefits is the sum of all the additional benefits to the systems for which the recommendation is applicable.

The benefit analysis did not aim to provide an accurate estimate of the added benefits of the different recommendations. It was rather used to provide an indication of the order of magnitude of additional potential benefits of the ITS due to the recommendations. The following scale was used to rate the order of magnitude of additional benefits:

- 0 : an estimated potential benefit of around 0-10 mln $€$

- +: an estimated potential benefit of around 10-100 mln $€$

Table 2 Parameters of assumed effectiveness of recommendations

\begin{tabular}{|c|c|c|c|c|c|c|}
\hline \multirow{2}{*}{$\begin{array}{l}\text { Effectiveness } \\
\text { area }\end{array}$} & \multirow[t]{2}{*}{ CBA parameter } & \multicolumn{5}{|c|}{ Impact of effectiveness levels in removing barriers } \\
\hline & & None & Very small & Small & Average & Large \\
\hline Safety & $\begin{array}{l}\text { Safety } \\
\text { Effectiveness }\end{array}$ & $100 \%$ & $102.5 \%$ & $105 \%$ & $110 \%$ & $120 \%$ \\
\hline Comfort & $\begin{array}{l}\text { Comfort } \\
\text { Effectiveness }\end{array}$ & $100 \%$ & $102.5 \%$ & $105 \%$ & $110 \%$ & $120 \%$ \\
\hline Mobility & $\begin{array}{l}\text { Mobility } \\
\text { Effectiveness }\end{array}$ & $100 \%$ & $102.5 \%$ & $105 \%$ & $110 \%$ & $120 \%$ \\
\hline Penetration rate & $\begin{array}{l}\text { Penetration } \\
\text { scenario }\end{array}$ & medium & $\begin{array}{c}1 / 8 \text { benefits of Large } \\
\text { effectiveness }\end{array}$ & $\begin{array}{c}\text { 1/4 benefits of Large } \\
\text { effectiveness }\end{array}$ & $\begin{array}{c}1 / 2 \text { benefits of Large } \\
\text { effectiveness }\end{array}$ & $\begin{array}{c}\text { Difference } \\
\text { high-medium scenario }\end{array}$ \\
\hline Costs & $\begin{array}{l}\text { Cost reduction } \\
(2020,2030)\end{array}$ & $(30 \%, 70 \%)$ & $(31.75 \%, 72.75 \%)$ & $(32.5 \%, 75 \%)$ & $(35 \%, 80 \%)$ & $(40 \%, 90 \%)$ \\
\hline
\end{tabular}


- ++: an estimated potential benefit of around $100 \mathrm{mln} €-1$ bn $€$

- +++: an estimated potential benefit of around 1-2.5 bn $€$

- ++++: an estimated potential benefit more than 2.5 bn $€$

An example of the calculations for the benefit analysis is provided in Box 1 below.

4. Final ranking of recommendations. The results of the benefit analysis, along with those of the qualitative analysis served as inputs for the Multi-Criteria Analysis (MCA). The MCA aimed to provide the final ranking of the 13 main recommendations. The MCA took into account four criteria: ease of implementation, time horizon, costs and potential benefits. Ease of implementation was divided into three categories: Easy, Medium and Challenging. These have a respective value of 3, 2 and 1 in the MCA. Similar scores were used for the two other (qualitatively assessed) criteria: time horizon and costs. The potential benefits of the recommendation were considered as the most important criterion, and therefore $50 \%$ of the scores were allocated to this aspect. The other criteria were considered equally relevant, therefore the remaining $50 \%$ of the scores were equally divided over these three aspects. This means that the maximum of the sum scores on the criteria ease of implementation, time horizon and costs is 9 .

The potential benefits criterion has four categories valued 1 to 4 in the MCA, based on the "+" to " $++++"$ scores. These values are multiplied by 2.25 in the overall score, to resemble that $50 \%$ of the scores come from potential benefits. In this way a maximum score on potential benefits (4 is the maximum value, multiplied by a weight of 2.25 equals 9 ) equals a maximum score on the remaining criteria ( 3 criteria with maximum value 3 also equals 9).

For example, imagine a recommendation with ease of implementation (medium), time horizon (long), costs (low) and potential benefits $(++)$. The weighted sum is then $2 * 1+1 * 1+$ $3 * 1+2 * 2.25=10.5$. The weighted sum of these criteria was then taken to be the overall score, ranking the first 13 recommendations. The various criteria scores and overall scores used in MCA are shown in Table 4.

\section{General recommendations}

\subsection{Analysis of the 10 its systems}

The potential barriers for widespread use of the ITS systems were identified by use of expert judgement of the consortium members, as well as recommendations to overcome these barriers. This was done by system and by topic. The number of identified barriers and recommendations are presented in Table 5.

As an example, Table 6 presents some barriers regarding technical performance of Blind Spot Detection (BSD) together with the classification on preliminary estimate on the ease to overcome the barrier.

Similarly, Table 7 outlines some recommendations regarding technical performance of Blind Spot Detection (BSD) together with the classifications (ease of implementation, time

\section{Box 1 Example assessment and calculation}

The recommendation 'designing traffic light control depending on traffic demand, taking into account VRU needs' (R7) is used as an example. The waiting times for all users are expected to decrease, through optimisation of the traffic light control. The recommendation will logically affect the Intelligent Pedestrian Traffic signal (IPT) system positively. This is confirmed by the participants of the workshop held by the VRUITS project, as it scored highest in the topic HMI \& acceptance. In this case, IPT is the only ITS that is expected to be affected by the recommendation, however, most of the time, multiple ITS systems are affected by a recommendation. A qualitative assessment of the effects of the recommendation on the system has been made:

Safety: The number of red-light running accidents is expected to decrease as annoyance is decreased due to shorter waiting times. In addition, it indirectly decreases the number of annoyance related accidents. This all leads to a very small increase in safety.

Mobility: Is only marginally increased, due to optimised waiting times.

Comfort: Optimisation of algorithms increases comfort very little for all road users.

Penetration rate: Improvement of algorithms makes the system more mature and reliable, resulting in the ability to install these systems in a greater variety of (traffic) situations. Thus a small increase in penetration rate can be expected.

Cost: An accelerated deployment may reap economies of scale benefits, leading to a small increase in discount rate.

Recommendation R7 has a long time horizon and for that reason the effects are estimated to only take place beyond 2020. Linking this to Table 2, the parameter values are thus easily determined (Table 3).

The parameter values are then used in the CBAs. For Safety, Mobility and Comfort, this is done as described earlier, multiplying the respective benefits with the given percentage. The increase in penetration rates is calculated by using the figures for the medium-usage scenario in 2020 , and the high-usage scenario in 2030. In the end, the difference with the original CBA output and the new CBA output is multiplied with $1 / 4$ to account for the corresponding "small" effect. The original CBA output is $€ 160.9 \mathrm{mln}$, the new CBA output is $€ 897.3 \mathrm{mln}$. Note that this new output seems fairly large in comparison with the original, but that's because in this form it represents the benefits for a large increase in penetration rate. Multiplying the difference with $1 / 4$ gives the correct value (values in mln $€$ ): $\frac{1}{4} *(897.3-160.9)=184.1$. In this case, $1 / 4$ of the difference of the original CBA results and new one is between $€ 100 \mathrm{mln}$ and $€ 1 \mathrm{bn}$, meaning the potential benefits are in the '++' category. 
Table 3 CBA inputs

\begin{tabular}{lll}
\hline & \multicolumn{2}{l}{ Quantified Effect: CBA inputs } \\
\hline Recommendation effects & 2020 & 2030 \\
Safety & $100 \%$ & $102.5 \%$ \\
Mobility & $100 \%$ & $102.5 \%$ \\
Comfort (car drivers) & $100 \%$ & $102.5 \%$ \\
Penetration rates & medium & $1 / 4$ of high penetration \\
Costs & $30 \%$ & $75 \%$ \\
\hline
\end{tabular}

horizon, estimated effectiveness and estimated costs) and relevant stakeholders.

A selection of the most relevant recommendations was made starting with the list of 208 recommendations. This selection resulted in a list of 50 recommendations divided into 3 groups (recommendations regarding VRUs, vehicles and infrastructure) which were discussed and reviewed during the workshop.

\subsection{Workshop and questionnaire}

A workshop has been held with the main stakeholders, including representatives of VRU groups, local governments and municipalities. The 10 selected ITS systems were presented and the list of 50 recommendations was discussed in three sessions. The final list of 22 recommendations (Table 8) was defined based on the feedback and rankings collected during the workshop from the list of 50 recommendations. (While analysing the results of the workshop, the recommendations with an overall score lower than five points were removed from the list and the recommendations which were closely related, and/or overlapping with each other, were merged with each other.)

\subsection{Further analysis of recommendations}

In order to develop a good basis on which to identify the most promising of the twenty-two recommendations, a number of aspects of each recommendation were identified and investigated in more depth. These aspects are:

- Develop a more extensive description of the recommendation. What does it encompass? What needs to be taken into account when considering this recommendation?

- Identify which systems are affected by this recommendation. Some recommendations can affect several systems, such as integration of several functions into a single device.

- Identify which of the eight topics (technical performance, safety, mobility \& comfort effects, human-machine interface (HMI), acceptance, business models, implementation issues, legal and standardisation issues and finally privacy, data storage, ethical and moral issues) this recommendation relates to.
Table 4 Overview of values and weights used in MCA in step 1

\begin{tabular}{|c|c|c|c|}
\hline Aspect/Indicator & Weight & Categories & Value \\
\hline \multirow[t]{3}{*}{ Ease of implementation } & \multirow[t]{3}{*}{1} & Easy & 3 \\
\hline & & Medium & 2 \\
\hline & & Challenging & 1 \\
\hline \multirow[t]{3}{*}{ Time horizon } & \multirow[t]{3}{*}{1} & Short & 3 \\
\hline & & Medium & 2 \\
\hline & & Long & 1 \\
\hline \multirow[t]{3}{*}{ Costs } & \multirow[t]{3}{*}{1} & Low & 3 \\
\hline & & Medium & 2 \\
\hline & & High & 1 \\
\hline \multirow[t]{4}{*}{ Potential benefits } & \multirow[t]{4}{*}{2.25} & + & 1 \\
\hline & & ++ & 2 \\
\hline & & +++ & 3 \\
\hline & & ++++ & 4 \\
\hline
\end{tabular}

- Description of main implementation issues. Make the issues more specific for the involved stakeholders.

- Time horizon. Provide main reasons for the estimated time horizon. Provide a more detailed overview of the actions needed for each stakeholder. Provide a high level action plan.

- Identify the barriers addressed by the recommendation, and the effectiveness of the recommendation to address the barrier (low/medium/high). For each barrier, indicate the ease of overcoming the barrier.

- Describe the main cost categories and cost-related barriers, the magnitude of the costs, and the relevant stakeholders

An excellent example is recommendation 10 from Table 8, "To design traffic light control, depending on the overall traffic demand (both vehicles and pedestrians) to minimise traffic delays for all road users while taking into account VRU needs and policy goals for safety, throughput, emissions and mobility". In this case, further investigation of the recommendation, based on the pilot in Spain, revealed that unnecessary delay created by traffic light control focusing solely on the pedestrian flow could be inefficient, as traffic lights at a single intersection are often part of a larger traffic control system. The traffic control should be designed so that:

1. A complete traffic view is obtained, not only of vehicles but also of pedestrians and cyclists,

2. Balance the needs of the Vulnerable Road users and motorised traffic needs, and take into account policy goals for safety, throughput, emissions and mobility

Several critical issues are illustrated below. Firstly, the main implementation issues identified for Recommendation 10 are: 
Table 5 Number of barriers and recommendations identified by system

\begin{tabular}{lcc}
\hline ITS system & $\begin{array}{l}\text { Number of } \\
\text { barriers }\end{array}$ & $\begin{array}{l}\text { Number of } \\
\text { recommendations }\end{array}$ \\
\hline Blind Spot Detection (BSD) & 22 & 25 \\
Bicycle to Vehicle Communication (B2V) & 24 & 17 \\
Crossing Adaptive Lighting (CAL) & 20 & 18 \\
Green Wave for Cyclists (GWC) & 25 & 29 \\
Intersection Safety (INS) & 18 & 11 \\
Intelligent Pedestrian Traffic Signal (IPT) & 31 & 29 \\
Information on Vacancy on Bicycle Racks (IVB) & 18 & 18 \\
Pedestrian and Cyclist Detection System + Emergency Braking & 19 & 21 \\
$\quad$ PCDS + EBR) & & 16 \\
PTW Oncoming Vehicle Information System (PTW2V) & 13 & 24 \\
VRU Beacon System (VBS) & 23 & 208 \\
Total & 213 & \\
\hline
\end{tabular}

- Development of detection sensors and algorithms, which detect all road users waiting and crossing the intersection, and potentially also of road users approaching the crossing.

- Research on algorithm development, dependent on traffic demand by all streams, needs to take place. Representatives of the special user groups should be involved in the algorithm development. These developments need to take into account that some countries use detectors for dynamic traffic light signal cycles.

- Traffic control suppliers need to be part of the development process and see a business case for implementation.

- Road authorities and governments need to pay for the implementation of these algorithms for their traffic lights.

An example of a barrier identified for Recommendation 10 is a user acceptance barrier: the system might cause slight annoyance to car drivers since prioritisation of pedestrians may lead to longer waiting times for cars (especially during rush hour). This could increase the red light running of cars. The annoyance caused to car drivers for giving prioritisation to pedestrians can be solved with a preliminary study of the intersection/area or environment in the traffic plan. In order to reduce waiting times for motorists, various technical developments could be envisaged: (i) pedestrian data could be entered into the traffic optimisation process (along with cyclists detected at the lights) to ensure optimum flow of all modes; (ii) pedestrian calls could be cancelled if the pedestrian has already crossed the street during a gap in traffic, before the pedestrian green phase has appeared. The impact on flow and safety of both types of developments would need to be established.

The primary stakeholders for this action were identified as follows, with remarks regarding the costs and roles:

- The EC: possibly involved in funding activities due to priority on Vulnerable Road Users and on Smart Cities (promote soft mode use, reducing emissions, promoting healthy lifestyles);

- Road authorities and governments should be involved in testing activities and need to pay for the implementation of these algorithms for their traffic lights;

- Research institutes: developing and critically evaluating the findings of the tests and ensure data collection during testing;
Table 6 Barriers regarding technical performance of Blind Spot Detection (BSD) together with the classification on preliminary estimate on the ease to overcome the barrier

\begin{tabular}{ll}
\hline Potential barriers & $\begin{array}{l}\text { Preliminary estimate on the ease } \\
\text { to overcome the barrier }\end{array}$ \\
\cline { 2 - 3 } & Easy $\quad$ Medium Challenging \\
\hline $\begin{array}{l}\text { Systems using camera may not work in poor lighting and adverse } \\
\text { weather conditions }\end{array}$ & $\mathrm{X}$ \\
$\begin{array}{l}\text { Blind spot detection for Powered Two Wheelers has other requirements } \\
\text { (other blind spots) than for non-motorised VRUs }\end{array}$ \\
$\begin{array}{l}\text { Currently there are different versions of the system on the market, } \\
\text { some of them requiring equipment on bicycles }\end{array}$ \\
$\begin{array}{l}\text { Detection rate for pedestrians (and cyclists) is still quite low, at 77\%. } \\
\text { This needs to increase. }\end{array}$ \\
\hline
\end{tabular}




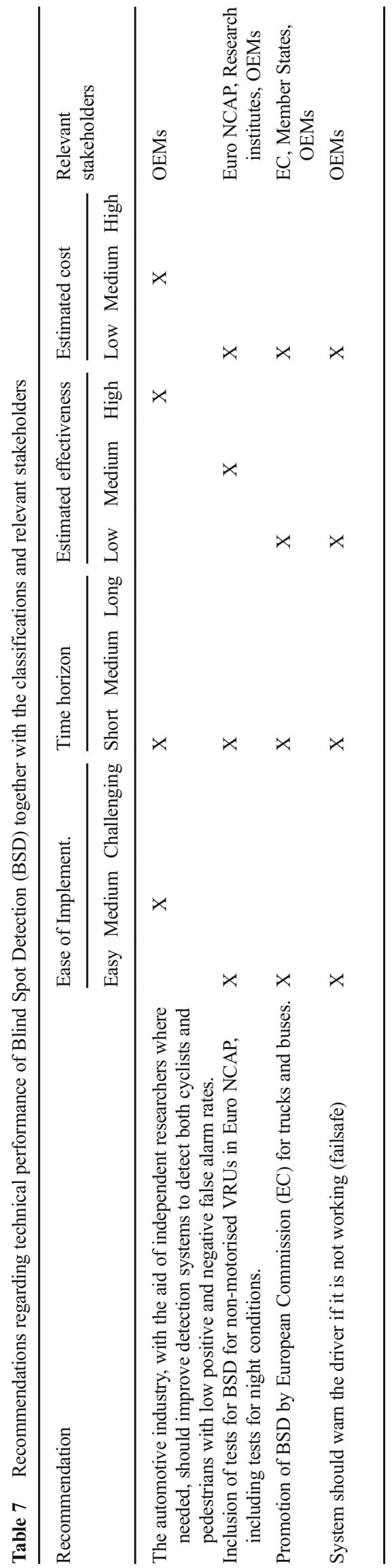

- Technical partners, system suppliers, sensor developers: developing and providing equipment for the field tests; and

- Representatives of user groups: ensure the needs of the specific groups are met by the innovation.

After the qualitative analysis of the 22 recommendations, a quantitative analysis was carried out for the main recommendations [8]. Eleven of these main recommendations were selected based on the overall scores at the workshop and the questionnaire. For the aspects "safety, mobility and comfort" (R5) and "privacy, data storage, ethical and moral issues" (R13), which were not covered by the selected recommendations, the recommendations R5 and R13, which scored best for these topics in the workshop and questionnaires were added. Table 9 provides an overview of the recommendations selected for quantitative analysis, together with the systems affected by these recommendations.

The affected systems, as shown in Table 9, have a central role in monetising the added benefits of carrying out the recommendations.

Table 10 shows the results of the quantitative analysis (based on calculations Ecorys (2016)). For more information on how these benefits are calculated, see Box 1 for an example or [8].

The recommendations that score best are research on VRU detection (R1) and research on road user behaviour (R2), both of which are research based. The added benefits are estimated to be over $€ 2.5$ billion (bn), with INS and PCDS + EBR as systems that increase their effectiveness the most. Similar results are seen for other recommendations, often with INS and PCDS + EBR contributing with positive values to the total impact. In addition, high positive effects are seen in PTW2V, contributing to higher total scores of a few recommendations. Recommendations with an impact between $€ 1$ bn and $€ 2.5$ bn are functionality integration (R11) research strategies for warning road users (R3), involve testing (R6 and R9), standardisation (R8) and privacy (R13).

\subsection{Final ranking of recommendations}

The results of the qualitative and quantitative analysis were used as input for a MCA to rank the 13 main recommendations. Table 11 presents an overview of the MCA results for the 13 main recommendations (based on calculations carried out by Ecorys). For more information on how the MCA was carried out, refer further to [8].

The results show that the highest scoring recommendations are all related to research activities and technical improvement. The fourth highest scoring recommendation is related 
Table 8 List of 22 recommendations

\section{No Recommendations}

Research for the improvement of VRU detection accuracy and classification for in-vehicle and infrastructure systems

2 Research for improving location accuracy of devices for VRUs, e.g. where GPS functions poorly, such as smart phones or devices for VRU vehicles, e.g. through information fusion with other sensors such as cameras

3 Research on strategies for warning road users to make the system more efficient and less annoying

4 Development of reliable low-latency wireless communication components (ITS-G5 or LTE), which are suitable for integration in VRU vehicles (bicycles, motorcycles) and smart phones

5 Research on road users' behaviour in traffic, especially of pedestrians (children) and prediction of intentions; including improved dynamics modelling of VRUs in traffic.

$6 \quad$ Research on optimal and efficient user interface

7 Reliability considerations (including harsh weather conditions and light pollution) should have a standard place in procurements of infrastructure related systems

8 Assessment of the possible effects of a potential mandatory use of the systems on long-term acceptance in different member states.

9 Testing of the systems in controlled situations and simulator testing to increase understanding of the interaction of different categories of VRUs with the systems

10 To design traffic light control, depending on the overall traffic demand (both vehicles and pedestrians) to minimise traffic delays for all road users while taking into account VRU needs and policy goals for safety, throughput, emissions and mobility

11 Standardisation of the functionalities of the systems, including VRU related cooperative systems and communication between VRUs and vehicles, that are required to enable interoperability

12 Creating field tests and system evaluations to provide more evidence on benefits, limitations and costs of ITS systems for VRU's.

13 Starting implementation with local I2VRU systems providing benefits to early adopters.

14 Development of low-cost high-quality technical solutions for VRU sensing systems for both vehicles and infrastructure

15 Traffic signal systems for intersections should be able to adapt to different environments in order to accommodate for different traffic management schemes and different environmental conditions

16 Integration of different (cooperative) functionalities in a single device

17 Liability issues should be governed prior to deployment of the systems and further investigated

18 Guidelines/ regulations are needed on the use of mobile devices on bicycles to avoid distraction

19 Development of guidelines and standardisation regarding fail safe operation (informing road users of nonor mal-functioning of the system).

20 Regulation about signal timing changes needs to be updated, to accommodate sensor based systems.

21 Create legislation that all trucks and buses should have blind spot detection systems with sensors back and at both sides

22 Ensure that privacy of all road users is guaranteed through development and use of proper procedures for data exchange, data collection and storage, and use of collected data to privacy aspects and data storage. The next two recommendations with 12.5 overall points, both score lower on potential benefits, but score relatively high on the other aspects, such as short time horizon for carrying out the recommendation. The recommendation concerning standards scored lower (R8) due to the high costs, while the recommendation concerning integration in one system scored lower due to the challenges experienced in implementation. The recommendations regarding testing of systems (R6 and R9) scored lower due to the relative high costs and challenging implementation. Recommendations regarding specific systems (R7 on IPT and R12 on BSD) score quite low, because they only concern one ITS system.

\section{Overview of the selected recommendations}

This section describes the recommendations with the highest scores (Table 11) in more detail.

Several of the recommendations are related to research to improve the performance of the systems, i.e. to determine reliably that there is a risk for an imminent collision and to decide to warn the driver or take a corrective action. In order to achieve a high performance, a high accuracy of the detection of the VRU and correct classification (R1) is needed, as well as an accurate prediction of the behaviour of the VRUs and their trajectories (R2), and as efficient warning strategies 
Table 9 Final list of 13 recommendations which were selected for Benefit Analysis (with new numbers)

\begin{tabular}{|c|c|c|}
\hline No & Recommendations & Systems affected \\
\hline $\mathrm{R} 1$ & $\begin{array}{l}\text { Research for the improvement of VRU detection accuracy and } \\
\text { classification for in-vehicle and infrastructure systems }\end{array}$ & INS, BSD, PCDS + EBR, VBS \\
\hline $\mathrm{R} 2$ & $\begin{array}{l}\text { Research on road users behaviour in traffic, especially of } \\
\text { pedestrians (children) and prediction of intentions; including } \\
\text { dynamics modelling of VRUs }\end{array}$ & INS, PCDS + EBR, VBS \\
\hline $\mathrm{R} 3$ & $\begin{array}{l}\text { Research on strategies for warning road users to make the system } \\
\text { more efficient and less annoying }\end{array}$ & $\begin{array}{l}\text { BCD, PCDS, VBS, INS, B2V, } \\
\text { PTW2V }\end{array}$ \\
\hline R4 & $\begin{array}{l}\text { Research on optimal and efficient user interface design (e.g. } \\
\text { information presentation, less distraction, reduced workload, } \\
\text { integration of user interface in bicycle, helmet, vest etc.). }\end{array}$ & $\begin{array}{r}\text { PTW2V, B2V, GWC, BSD, } \\
\text { PCDS + EBR, INS, VBS }\end{array}$ \\
\hline R5 & $\begin{array}{l}\text { Reliability considerations (including harsh weather conditions and } \\
\text { light pollution) should have a standard place in procurements of } \\
\text { infrastructure related systems }\end{array}$ & GWC, INS, CAL \\
\hline R6 & $\begin{array}{l}\text { Testing of the systems in controlled situations and simulator } \\
\text { testing to increase understanding of the interaction of different } \\
\text { categories of VRUs with the systems }\end{array}$ & $\begin{array}{l}\text { GWC, B2V, PTW2V, IPT, BSD, } \\
\text { PCDS + EBR, VBS, INS }\end{array}$ \\
\hline R7 & $\begin{array}{l}\text { To design traffic light control, depending on the overall traffic } \\
\text { demand (both vehicles and pedestrians) to minimise traffic } \\
\text { delays for all road users while taking into account VRU needs } \\
\text { and policy goals for safety, throughput, emissions and mobility }\end{array}$ & IPT \\
\hline $\mathrm{R} 8$ & $\begin{array}{l}\text { Standardisation of the functionalities of the systems, including } \\
\text { VRU related cooperative systems and communication between } \\
\text { VRUs and vehicles, that are required to enable interoperability }\end{array}$ & $\begin{array}{l}\text { B2V, GWC, PTW2V, VBS, BSD, } \\
\text { PCDS + EBR, CAL }\end{array}$ \\
\hline R9 & $\begin{array}{l}\text { Creating field tests and system evaluations to provide more } \\
\text { evidence on benefits, limitations and costs }\end{array}$ & $\begin{array}{l}\text { BSD, PCDS + EBR, CAL, IPT, } \\
\text { IVB }\end{array}$ \\
\hline $\mathrm{R} 10$ & $\begin{array}{l}\text { Traffic signal systems for intersections should be able to adapt to } \\
\text { different environments in order to accommodate for different } \\
\text { traffic management schemes and different environmental } \\
\text { conditions }\end{array}$ & IPT \\
\hline $\mathrm{R} 11$ & $\begin{array}{l}\text { Integration of different (cooperative) functionalities in a single } \\
\text { device }\end{array}$ & GWC, B2V, PTW2V, IVB \\
\hline $\mathrm{R} 12$ & $\begin{array}{l}\text { Create legislation that all trucks and buses should have blind spot } \\
\text { detection systems with sensors back and at both sides }\end{array}$ & BSD \\
\hline $\mathrm{R} 13$ & $\begin{array}{l}\text { Ensure that privacy of all road users is guaranteed through } \\
\text { development and use of proper procedures for data exchange, } \\
\text { data collection and storage, and use of collected data }\end{array}$ & $\begin{array}{l}\text { B2V, GWC, PTW2V, IPT, IVB, } \\
\text { VBS }\end{array}$ \\
\hline
\end{tabular}

(R3) and efficient user interfaces (R4) to achieve the desired reaction of the road user.
Significant R\&D has been performed on detecting pedestrians by vehicle sensors [9], much less research has been

Table 10 Results quantitative analysis

\begin{tabular}{|c|c|c|c|c|c|c|c|c|c|c|c|c|c|}
\hline \multirow[b]{2}{*}{ System } & \multicolumn{13}{|c|}{ Recommendation } \\
\hline & $\mathrm{R} 1$ & $\mathrm{R} 2$ & $\mathrm{R} 8$ & $\mathrm{R} 6$ & R13 & R9 & R11 & R3 & $\mathrm{R} 5$ & $\mathrm{R} 4$ & $\mathrm{R} 10$ & $\mathrm{R} 7$ & $\mathrm{R} 12$ \\
\hline Total impact & ++++ & ++++ & +++ & +++ & +++ & +++ & +++ & +++ & ++ & ++ & ++ & ++ & + \\
\hline $\mathrm{B} 2 \mathrm{~V}$ & - & - & ++ & + & ++ & - & + & + & - & + & - & - & - \\
\hline BSD & + & - & + & ++ & - & + & - & + & - & + & - & - & + \\
\hline CAL & - & - & ++ & - & - & ++ & - & - & ++ & - & - & - & - \\
\hline GWC & - & - & + & 0 & 0 & - & 0 & - & 0 & 0 & - & - & - \\
\hline INS & ++++ & +++ & - & ++ & - & - & - & 0 & ++ & $+/++$ & - & - & - \\
\hline IPT & - & - & - & + & 0 & ++ & - & - & - & - & ++ & ++ & - \\
\hline IVB & - & - & - & - & 0 & 0 & 0 & - & - & - & - & - & - \\
\hline $\mathrm{PCDS}+\mathrm{EBR}$ & ++++ & +++ & ++ & + & - & ++ & - & ++ & - & 0 & - & - & - \\
\hline PTW2V & - & - & ++ & +++ & ++ & - & +++ & + & - & ++ & - & - & - \\
\hline VBS & + & + & ++ & ++ & ++ & - & - & + & - & ++ & - & - & - \\
\hline
\end{tabular}


Table 11 Overview of MCA results and ranking of 13 main recommendations

\begin{tabular}{|c|c|c|c|c|c|}
\hline Recommendation & Ease of implement. & Time Horizon & Estimated Costs & Potential Benefits & Overall score \\
\hline R2 Research on road users behaviour & medium & medium & medium & ++++ & 15 \\
\hline R1 Research on improving VRU detection accuracy & medium & medium & high & ++++ & 14 \\
\hline R3 Research on warning strategies & medium & medium & medium & +++ & 12.75 \\
\hline R13 Guarantee privacy of all road users & medium & medium & medium & +++ & 12.75 \\
\hline R5 Reliability criteria in procurement & easy & short & medium & ++ & 12.5 \\
\hline R10 Traffic light control tailored to environment & medium & short & low & ++ & 12.5 \\
\hline R8 Standardisation of functionalities & medium & medium & high & +++ & 11.75 \\
\hline R11 Functionalities integration in one device & challenging & medium & medium & +++ & 11.75 \\
\hline R6 Testing of systems in controlled situations & challenging & medium & high & +++ & 10.75 \\
\hline R9 Field tests and system evaluations & challenging & medium & high & +++ & 10.75 \\
\hline R4 Design of optimal and efficient user interface & medium & medium & high & ++ & 9.5 \\
\hline R7 Traffic light control based on overall demand & medium & medium & high & ++ & 9.5 \\
\hline R12 Legislation of blind spot detection for trucks & medium & medium & low & + & 9.25 \\
\hline
\end{tabular}

conducted regarding cyclists [10] and other VRUs. Work on accurate prediction of the behaviour of VRUs and their trajectories has been performed by Brunsmann et al. [11] and Köhler et al. [12]. The Euro NCAP rating system includes AEB systems for specific scenarios for pedestrians since 2016 and from 2018 onwards, will include both cyclists as well as night and obscure lighting conditions [13], which will stimulate development and deployment in this field. Additional funding is required in order to stimulate the development of systems addressing a wide range of road user types in challenging urban settings and environmental conditions.

User interface design, together with the comfort levels of the user, are crucial in influencing the use of the systems and the compliance with the advice provided by the system. The user interface should be designed to receive a correct and timely response of the road users addressing aspects such as comprehensible information presentation, minimum distraction and a reduced workload. Guidelines have been developed for user interfaces for drivers, e.g. [14, 15]. Aspects which require further research include integration of ITS and warnings to bicycles, helmets, vests, and the optimal way to provide warnings to the different road users.

The system warnings should initiate the desired reaction by the road user without causing annoyance, which can occur in case of too frequent low risk warnings. For vehicle drivers, research on warning strategies has been performed for ADAS systems [16, 17]. As the amount of assisting systems increases and hence potential warnings, guidelines are required for developers on the optimal timing and warning modes for drivers and other road users, to achieve an efficient reaction and increased system acceptance. Efficient warning strategies will have a positive effect on safety, and result in a small increase of penetration rate.
Recommendations R6 and R9 are both related to testing. Testing both in controlled situations and in a simulator testing is needed in order to increase the understanding of the interaction of different categories of VRUs - including elderly persons, people with limited mobility and vehicle drivers with the systems (R6). Large scale field tests and system evaluations are needed to provide more evidence on benefits, limitations and costs of the systems (R9), as well as provide information on the long-term effects of the systems on the behaviour of both VRUs and vehicle drivers. This will produce a more concrete understanding of the feasibility of installing an ITS. The costs and complexity of field tests and system evaluations are often too large for investors to bear alone, and therefore require collaboration between stakeholders from different sectors and domains.

Two recommendations relate to signalised intersections: "Design of traffic light control systems which take the whole traffic demand of both vehicles and VRUs into account" (R7) and "Traffic signal systems for intersections should be able to adapt to different environments in order to accommodate for different traffic management schemes and different environmental conditions" (R10). Technologies such as big data and IoT allow for a real-time view of all road users approaching, waiting and crossing the intersection. This allows for the optimisation of traffic light phases for all road users, taking into account the VRU needs and policy goals for throughput, emissions and mobility, as well as avoiding unnecessary waiting times for road users.

Standardisation of the functionalities of the systems, including VRU related cooperative systems and communication between VRUs and vehicles, is required to enable interoperability (R8). For C-ITS systems, current message implementations, such as CAM and DENM do not take VRUs into account in a structured way. Recently, work on this subject has started in ETSI with the study on 
VRU use cases and standardisation perspectives (TR 103300). Further progress of this standardisation work for ITS systems for VRUs and underlying message sets and communication networks needs to take place in the upcoming years based on new insights, applications, and technologies.

Standardisation is a prerequisite to make the integration of different (cooperative) functionalities in a single device (R11) possible. The ITS applications for VRUs can either be deployed as apps in smartphones or on dedicated devices, which are integrated in the VRU vehicles, such as electric bikes, mopeds and motorcycles. The integration of communication equipment in VRU vehicles provides challenges, including limited cost, size and power consumption of the devices. Research and development has to address the physical integration of the devices in the vehicles, including the antenna design and radiation patterns for optimal performance of the devices. In case smartphones are used, the smartphone has to sense the context of the user, e.g. is the user walking outdoors or indoors, sitting in a bus, etc.

Privacy is critical in ITS systems, and is a major barrier to the deployment of systems. This is especially the case for CITS systems [18], where users broadcast data on their location. The C-ITS Platform addresses privacy and security, mainly for vehicle systems, but needs to be further developed to include VRU applications. Proper procedures for data exchange, data collection and storage, and the use of collected data (R13) have to be developed to guarantee the privacy of all road users.

Recommendation R12 relates to the blind-spot detection system. Legislation has to be implemented enforcing all trucks and buses to have blind spot detection systems with sensors at the back and at both sides of the vehicle.

For all infrastructure related systems, reliability considerations (including harsh weather conditions and light pollution) should have be accounted for in procurement procedures (R5). Governments should therefore include a comprehensive set of rules regarding a wide range of aspects ensuring that the deployed system works reliably in all environmental conditions and mitigates negative external effects (e.g. light pollution).

\section{Pros and cons of methodology}

An overview of the pros and cons of the methodology for identifying and ranking recommendations for actions supporting ITS developments for VRUs can be provided by using the experiences in the VRUITS project. In addition, some guidelines will be provided for exploiting the methodology in future projects.

\subsection{Pros of the methodology}

- The main advantage of the methodology is that it provides a filtering process that can be applied to shorten a long list of a very broad range of recommendations which have been defined based on qualitative information and to arrive at a short list of recommendations, by exploiting a combination of qualitative and quantitative information. The stepwise selection process of relevant recommendations ensured that most attention and in-depth analysis is focused to the most promising recommendations.

- The methodology uses available results from earlier work done in the VRUITS project, such as the CBA calculations. Detailed interim results of the various steps provide relevant information which can be further used in the future work.

- The adopted stepwise methodology included multiple validation checks with experts. Therefore, the methodology offered sufficient possibilities for feedback and review to improve the interim results. Furthermore, the workshop offered the possibility to discuss the interim results with the main stakeholders and to take their suggestions into account.

- Quantification using classes of potential benefits provided an indication of the order of magnitude of the expected effects, despite the limited available quantitative information. The potential benefits were presented in this way in order to avoid a false expression of accuracy, which would be the case if we would present potential benefits in euros.

\subsection{Cons of the methodology}

- It is a cumbersome methodology with a significant number of steps, which need to be carried out to define a final short list of recommendations.

- A significant amount of time is needed to apply the methodology. Furthermore, the success of the execution of the methodology depends on the willingness of external experts to contribute to the process. The consulted experts must allocate sufficient time to the consultation and they need to be well prepared in order to fully understand the questions and provide relevant inputs.

- There are some subjective elements in the methodology, such as quantifying the impacts using categories very small/small/average/large and when defining the weights in the MCA used for ranking the recommendations. The risk of subjective elements was minimised by involving stakeholders and experts in the process of validating the interim results. 
- Results of combining multiple recommendations during the benefit analysis of the main recommendations was not investigated in the VRUITS project. However the methodology is flexible enough to be adapted to take this into account, for example, by handling the combination of recommendations as a new recommendation and to carry out the same benefit analysis as has been used for a single recommendation.

\subsection{Guidelines for using the methodology in future projects}

The methodology can be applied in future projects when CBA calculations are available, or can be performed for the ITS systems that are examined. The methodology can also be used where limited quantitative inputs are available. These can be used to provide detailed quantified benefits.

An alternative application of this methodology is to use another, less complicated way to estimate the classes of potential benefits by exploiting only qualitative input data. This would render the methodology less time consuming. However if the analysis is done in a qualitative way, the results will probably be less robust than when using the quantitative input data.

This methodology can also be more easily applied when it is possible to collect and use additional quantitative data in a more limited scale than in the VRUITS project for the calculation of the potential costs and benefits of applying the ITS. This could be the case when referring to a regional and local level, where more detailed quantitative information is likely to be available.

\section{Conclusions}

\section{A wide range of different type of recommendations direct- ed to different stakeholders were identified during the assessment.}

In order to achieve the full potential of the ITS systems at EU level, a wide range of barriers have to be overcome, including technical issues (detection inaccuracy, uncertainty in road user trajectories), user acceptance issues (high level of unnecessary warnings, lack of suitable user interface), business models (high costs, chicken-egg problems) and legal issues (privacy, current legislation). In order to address these barriers, a large set of recommendations has been identified, covering a wide range of potential measures, including research activities to technical aspects, field tests and simulation, behaviour and usage aspects. Cooperation of a wide range of stakeholders is critical for implementing the recommendations: European Commission, national authorities, various industries, user organisations and research organisations all play a role. Market related aspects and business models should also be investigated.

\section{Focus should be on the implementation of the 13 main recommendations.}

Based on the results of the analysis carried out, the focus should be on implementing the thirteen main recommendations. The recommendations regarding procurement (R5) and system design of traffic control systems (R10) can almost be directly taken forward. The technical recommendations regarding road user modelling (R1) and location accuracy (R2) are critical, as they allow the systems to more accurately predict potential collisions. Improved technical performance increases user acceptance by reducing "nuisance" alerts and enables increased efficiency in warning the driver or taking corrective actions. Major issues are the costs of the systems and privacy related issues. Field tests and large pilots which study how road users interact with the systems and demonstrate the benefits of the systems are needed. In order to implement the suggested recommendations successfully, all stakeholders have to be involved. The implementation and time planning of the other recommendations should be further discussed with the main stakeholders. A relevant aspect when discussing implementation is also the stability and safety of each ITS system, which can be addressed by looking at the possibility of malfunctions, consequences of ITS failure and potential maintenance costs. OEMs and infrastructure system providers should be stimulated to implement the sensors system and collision prevention tools. Authorities should be stimulated to deploy the systems, and road users encouraged to use the devices and systems which have been designed to improve their safety.

\section{Benefits of implementation of recommendations can be in the order of billion(s) euros.}

Most of the recommendations address several ITS systems, including all in-vehicle systems. Recommendations affecting multiple ITS systems can have large potential benefits. In the Benefit Analysis, calculations of five factors have been taken into account: safety, mobility, comfort effectivity, penetration rates and costs. Having done this on multiple systems for 13 recommendations, it can be concluded that especially increasing the penetration rate (i.e. number of users European-wide) can greatly contribute to the total benefits.

4. Main challenges are cooperation of different stakeholders, different environments, long time horizon for implementation and high implementation costs of many recommendations.

Most of the recommendations include challenges at a technical level or requiring the collaboration of a wide set of 
stakeholders, which cannot be resolved within a short time horizon (shorter than 5 years). A similar conclusion is made in relation to costs: only a few solutions have low costs, and the most effective recommendations are less likely to have low costs.

Acknowledgements The research leading to these results has received funding from the European Union's Seventh Framework Programme for research, technological development and demonstration under grant agreement $n^{\circ} 321586$.

Open Access This article is distributed under the terms of the Creative Commons Attribution 4.0 International License (http:// creativecommons.org/licenses/by/4.0/), which permits unrestricted use, distribution, and reproduction in any medium, provided you give appropriate credit to the original author(s) and the source, provide a link to the Creative Commons license, and indicate if changes were made.

\section{References}

1. European Commission (2010) DIRECTIVE 2010/40/EU on the framework for the deployment of Intelligent Transport Systems in the field of road transport and for interfaces with other modes of transport

2. European Road Safety Observatory (2016) Traffic safety - basic facts 2016, Urban areas

3. Scholliers J, Bell D, Morris A, García Meléndez AB, Perez OM (2016) Improving safety and mobility of vulnerable road users through ITS applications. In: Yannis G, Cohen S (eds) Traffic safety. John Wiley \& Sons, Inc., Hoboken, pp 251-269

4. Scholliers J, van Noort M, Johansson C, Mans D, Silla A, Bell D, Hancox G, Leden L, Giannelos I, Bax B, Malone K (2016) Impact assessment of ITS applications for vulnerable road users. Trans Res Procedia 14(2016):4515-4524

5. Kulmala R (2010) Ex-ante assessment of the safety effects of intelligent transport systems. Accid Anal Prev 42:1359-1369
6. van Noort, M., Malone, K., Silla, A., Leden, L., Rämä, P., Innamaa, S. Johansson, C., Bell, D., Giannelos, I., Mans, D., van Schijndel, M., Morris, A. 2014. Assessment methodology. VRUITS project deliverable D2.2.

7. Scholliers, J., van Dam, E., Malone, K., Heuven, D., de Gelder, E., Brantova, V., Gonzalez Luna, J, Martin Fernandez, S., Martin Perez, O (2016) Evaluation results, VRUITS project deliverable D5.3

8. Van Breemen R, Giannelos I, Malone K, Mans D, Räma P, Scholliers J, Silla A (2016) Recommendation for actions at EU level and their assessment. VRUITS Deliverable D6:2

9. Gerónimo D, López A, Sappa A, Graf T (2010) IEEE. Trans Pattern Anal Machine Intel 32(7):1239-1258

10. Lee Y, Kim T, Shim J (2016) Two-wheeler detection algorithm research based on volumetric local binary pattern using projection vector features. Int J Software Eng Appl 10(5):143-156

11. Brunsmann U, Seeliger F, Wertheimer R (2013) Ko-PER spezifische Nachrichteninhalte (öffentliche Fassung). Forschungsinitiative Ko-FAS, $\mu$ MS 2:720-722

12. Köhler S, Goldhammer M, Bauer S, Doll K, Brunsmann U, Dietmayer K (2012) Early detection of the Pedestrian's intention to cross the street, $15^{\text {th }}$ Int IEEE Conf on ITS, Anchorage, USA, 16-19 Sept 2012

13. Euro NCAP (2014) Euro NCAP's road map 2020 - the next steps for vulnerable road user AEB assessment. ASPECCS Final Workshop, Aschaffenburg, Germany 1(7):2014

14. European Commission (2008) Commission recommendation of 26 may 2008 on safe and efficient in-vehicle information and communication systems: update of the European statement of principles on human-machine interface. Off J Eur Union 12(8):2008

15. Car Connectivity Consortium (2015) MirrorLink SpecificationApplication requirements for Drive Certification, version 1.0.23(CCC-RQ-005)

16. Ljung Aust M, Dombrovskis S (2013) Understanding and improving driver compliance with safety system feedback, $23^{\text {rd }}$ enhanced safety of vehicles conference. Seoul, Korea, pp 27-30

17. Johansson E (2013) Information. Warning and Intervention Strategies, InteractIVe Final Event, Aachen, Germany, pp 20-21

18. C-ITS Platform, Final report, January 2016 